PROCEEDINGS OF THE

AMERICAN MATHEMATICAL SOCIETY

Volume 131, Number 3, Pages 675-684

S 0002-9939(02)06843-0

Article electronically published on October 23, 2002

\title{
REDUCTIVE EMBEDDINGS ARE COHEN-MACAULAY
}

\author{
ALVARO RITTATORE \\ (Communicated by Dan M. Barbasch)
}

\begin{abstract}
In this paper we prove that in positive characteristics normal embeddings of connected reductive groups are Frobenius split. As a consequence, they have rational singularities and are thus Cohen-Macaulay varieties.

As an application, we study the particular case of reductive monoids, which are affine embeddings of their unit group. In particular, we show that the algebra of regular functions of a normal irreducible reductive monoid $M$ has a good filtration for the action of the unit group of $M$.
\end{abstract}

\section{INTRODUCTION}

Let $G$ be a complex connected reductive algebraic group and $X$ a complex spherical $G$-variety. In [1] it is proved that if $p$ is a prime number big enough, then the reduction $\bmod p$ of $X$ is Frobenius split and, in particular, has rational singularities. Thus, in characteristic zero $G \times G$-equivariant normal embeddings of $G$-where $G \times G$ acts by left and right multiplication - have rational singularities. However, some spherical varieties in characteristic $p>0$ are not Frobenius split; examples are quotients of a connected semi-simple group by non-reduced parabolic subgroupschemes (see [9]). In this work we show that, in arbitrary positive characteristic, if $G$ is reductive, then any normal $G$-embedding is Frobenius split, and as a consequence, it has rational singularities; moreover, we show that there exists a canonical Frobenius splitting. This can be done because of the good control of the geometry of these varieties, which allows us to follow the argumental lines used in the case of the complete symmetric varieties (see [15] and [2]). In particular, normal irreducible reductive monoids, i.e. algebraic monoids whose unit group is connected and reductive, have rational singularities. A first step in the resolution of this problem for reductive monoids can be found in [13, where it is shown that reductive monoids with unit group $\mathrm{Gl}_{2}(k), \mathrm{PGl}_{2}(k) \times k^{*}$ and $\mathrm{Sl}_{2}(k) \times k^{*}$ are Cohen-Macaulay varieties.

As an application, we generalize a result by Doty ([3]) about the existence of good filtrations for normal reductive monoids with one-dimensional center to arbitrary reductive monoids.

The author wishes to thank M. Brion for many useful remarks and suggestions.

Received by the editors September 28, 2000.

2000 Mathematics Subject Classification. Primary 14M17, $14 \mathrm{M} 05$.

Key words and phrases. Reductive embeddings, reductive monoids, Cohen-Macaulay varieties, spherical varieties.

This research was partially done during a stay at the Institut Fourier, Grenoble, France. 


\section{Preliminaries}

In this section we recall the basic facts about the classification of reductive embeddings - i.e. embeddings of a reductive group - to be used in this work.

Definition 1. Let $G$ be a connected reductive group over a field $k$ of arbitrary characteristic. A homogeneous space $G / H$ is spherical if there exists a Borel subgroup $B$ of $G$, such that $B H$ is open in $G$. If $X$ is a $G$-variety with an open orbit isomorphic to $G / H$, then $X$ is called a $G / H$-embedding. If moreover $X$ is normal, we say that $X$ is a spherical variety. We say that $X$ is simple if the action of $G$ over $X$ has only one closed orbit.

Note that $G \cong(G \times G) / \Delta(G)$, where $\Delta(G)$ is the diagonal in $G \times G$, is a $(G \times G)$ spherical homogeneous space. Thus, a $G$-embedding is a $(G \times G) / \Delta(G)$-embedding.

A reductive monoid is an algebraic monoid with unit group a reductive group. They are reductive embeddings; moreover we have the following:

Theorem 1 (14]). The irreducible reductive monoids are exactly the affine embeddings of reductive groups. The commutative irreducible reductive monoids are exactly the affine embeddings of tori.

Notation. From now on, we restrict ourselves to the study of normal reductive embeddings and irreducible normal reductive monoids, unless stated otherwise. All reductive groups are supposed to be connected.

Spherical varieties, and thus reductive monoids, are classified in combinatorial terms (cf. 14 for the case of reductive monoids and [8] for the general case of spherical varieties). For reductive monoids, this classification is dual to that made by Vinberg in 17] in characteristic zero, in terms of the decomposition of their algebra of regular functions for the action of the unit group.

We will use some properties of the classification of spherical varieties in order to describe the geometry of reductive embeddings, in particular in order to find a desingularisation for any reductive embedding, so we summarize it in order to fix notations. Let $G / H$ be a spherical homogeneous space, and $B$ be a Borel subgroup of $G$ such that $B H \subset G$ is open. We denote $k(G / H)^{(B)}$ to be the set of $B$ eigenvectors of $k(G / H)$, the field of rational functions of $G / H$, and $\Lambda_{G / H}$ to be the set of weights of $k(G / H)^{(B)}$. We consider the space $\operatorname{Hom}_{\mathbb{Z}}\left(\Lambda_{G / H}, \mathbb{Z}\right) \otimes \mathbb{Q}=$ $\mathcal{Q}(G / H)$; the restriction to $k(G / H)^{(B)}$ induces an injection of the set of $G$-invariant valuations of the field $k(G / H)$, into $\mathcal{Q}(G / H)$. Its image is a rational polyhedral cone, the valuation cone, denoted $\mathcal{V}(G / H)$. On the other hand, if we denote $\mathcal{D}(G / H)$ to be the set of the irreducible $B$-stable divisors of $G / H$, the colors, then there exists a map $\rho: \mathcal{D}(G / H) \rightarrow \mathcal{Q}(G / H)$.

To each simple spherical variety $X$ with open orbit $G / H$ corresponds a unique colored cone, namely a pair $(\mathcal{C}(X), \mathcal{F}(X))$ constructed as follows: let $Y$ be the unique closed $G$-orbit of $X$, and put $\mathcal{F}(X)=\{D \in \mathcal{D}(G / H) \mid \bar{D} \supset Y\}$. Let $\mathcal{B}(X)$ be the subset of $\mathcal{V}(G / H)$ consisting of the valuations associated to the irreducible $G$-stable divisors of $X$. Then $\mathcal{C}(X)$ is the strictly convex polyhedral cone generated by $\mathcal{B}(X)$ and $\rho(\mathcal{F}(X))$. This cone verifies that $\mathcal{C}(X)^{\circ}$, the relative interior of $\mathcal{C}(X)$, intersects the valuation cone.

Conversely, a pair $(\mathcal{C}, \mathcal{F})$ verifying the properties described above determines a unique $G / H$-spherical variety such that $(\mathcal{C}(X), \mathcal{F}(X))=(\mathcal{C}, \mathcal{F})$. Non-simple spherical varieties can be classified by means of colored fans, which are collections 
of colored cones with some compatibility restrictions. If the set of colors is empty, we say that the associated spherical variety is without colors, and we write $\mathcal{C}$ instead of $(\mathcal{C}, \emptyset)$ if no confusion is possible.

Finally, we recall that if $X$ and $X^{\prime}$ are spherical varieties with open orbits $G / H$ and $G / H^{\prime}$ respectively, dominant $G$-equivariant morphisms $\varphi: X \rightarrow X^{\prime}$ are in bijection with linear maps $\varphi_{*}: \mathcal{Q}(G / H) \rightarrow \mathcal{Q}\left(G / H^{\prime}\right)$ such that $\varphi_{*}\left(\mathcal{E}_{X}\right) \subset \mathcal{E}_{X^{\prime}}$, where $\mathcal{E}_{X}$ denotes the colored fan associated to $X$ (cf. [8]).

Notation. Let us fix some notations. Let $G$ be a reductive group, and let $T$ be a maximal torus of $G$. Let $B$ be a Borel subgroup containing $T$, and $B^{-}$its opposite Borel subgroup. We denote $\Xi(T)$ as the set of weights and $\Xi_{+}(T)$ the semigroup of dominant weights with respect to $B$. We denote $W$ to be the Weyl group associated to $T$, and $C=C(G)$ to be the Weyl chamber associated to $(B, T)$. We denote $\alpha_{1}, \ldots, \alpha_{l}$ and $\omega_{1}, \ldots, \omega_{l}$ to be the simple roots and fundamental weights associated to $(B, T)$, respectively.

Finally, we denote $\Xi_{*}(T)$ to be the set of one parameter subgroups (1-PS) of $T$. We identify $\Xi_{*}(T)$ with $\Xi(T)$ by means of a $W$-invariant scalar product $\langle\cdot, \cdot\rangle$, in such a way that $\left\langle\omega_{i}, \alpha_{j}^{\vee}\right\rangle=\delta_{i j}$.

From the Bruhat decomposition we get that the subvarieties $D_{i}=\overline{B s_{\alpha_{i}} B^{-}}$, $s_{\alpha_{i}} \in W$, the reflection associated to the simple root $\alpha_{i}, i=1, \ldots, l$, are exactly the irreducible $\left(B \times B^{-}\right)$-stable divisors of $G$ (the colors).

The combinatorial data associated to the spherical homogeneous space $G=$ $(G \times G) / \Delta(G)$ are the space $\mathcal{Q}(G)=\Xi(T) \otimes \mathbb{Q}$, with valuation cone $\mathcal{V}(G)=-C$, and colors $\alpha_{i}^{\vee}, i=1, \ldots, l([14])$.

\section{Frobenius SPlitting for $G$-Embeddings}

In this section we prove that any normal reductive embedding has a rational resolution, and thus is Cohen-Macaulay.

Definition 2. We say that a birational proper morphism $\varphi: X \rightarrow Y, X$ a irreducible smooth variety, is a rational resolution of $Y$ if:

$$
\begin{array}{lll}
\varphi_{*} \mathcal{O}_{X}=\mathcal{O}_{Y}, & R^{i} \varphi_{*}\left(\mathcal{O}_{X}\right)=0 & i>0, \\
\varphi_{*} \omega_{X}=\omega_{Y}, & R^{i} \varphi_{*}\left(\omega_{X}\right)=0 & i>0 .
\end{array}
$$

Here $\omega_{X}$ is the canonical sheaf of $X$, and $\omega_{Y}=i_{*} \omega_{Y_{\text {reg }}}$, where $i: Y_{\text {reg }} \hookrightarrow Y$ is the inclusion of the regular locus. If there exists a rational resolution for $Y$, then $Y$ is normal and Cohen-Macaulay.

Definition 3. Assume that $\operatorname{char} k=p>0$. If $X$ is a scheme over $k$, we denote $F$ to be the absolute Frobenius morphism. We say that $X$ is Frobenius split if the induced map $F: \mathcal{O}_{X} \rightarrow F_{*} \mathcal{O}_{X}$ is split, i.e. there exists a map $\sigma \in \operatorname{Hom}_{\mathcal{O}_{X}}\left(F_{*} \mathcal{O}_{X}, \mathcal{O}_{X}\right)$ such that $\sigma \circ F$ is the identity. We call $\sigma$ a (Frobenius) splitting. If $Y \subset X$ is a closed subscheme with associated ideal sheaf $\mathcal{I}_{Y}$, we say that $\sigma$ is compatible with $Y$ if $\sigma\left(F_{*} \mathcal{I}_{Y}\right) \subset \mathcal{I}_{Y}$

In order to find a Frobenius splitting for a given variety, we will use the following criterion:

Proposition 1 (12],[11]). Let $X$ be a complete smooth variety over a field $k$ of characteristic $p>0$. Then $\operatorname{Hom}_{\mathcal{O}_{X}}\left(F_{*} \mathcal{O}_{X}, \mathcal{O}_{X}\right) \cong F_{*} H^{0}\left(X, \omega_{X}^{1-p}\right)$. In particular, a section $\sigma \in H^{0}\left(X, \omega_{X}^{1-p}\right)$ induces a Frobenius splitting if and only if for some point 
$q \in X$ and some local system of coordinates $x=\left(x_{1}, \ldots, x_{N}\right)$ at $q$, the coefficient of $(x / d x)^{p-1}$ in the expansion of $\sigma$ at $q$ is non-zero.

First of all, we extend some known results about resolutions of singularities of $G$-embeddings to arbitrary characteristics.

Proposition-Definition ([4],[18],[15]). Let $G$ be a reductive group and $Z$ be its center. Then there exists a maximal simple projective embedding of $G_{a d}=G / Z \cong$ $\left(G_{a d} \times G_{a d}\right) / \Delta\left(G_{a d}\right)$. We call this embedding the wonderful embedding of $G_{a d}$ (or $G)$ and denote it as $\mathbf{X}$.

Let us summarize the properties of the wonderful embedding to be used in our work:

The wonderful embedding is a smooth variety.

If $C=C(G)$ notes the Weyl chamber of $G$ associated to a Borel subgroup $B$, then the colored cone associated to the wonderful embedding is

$$
(\mathcal{C}(\mathbf{X}), \mathcal{F}(\mathbf{X}))=\left(\mathcal{V}\left(G_{a d}\right), \emptyset\right)=-C\left(G_{a d}\right) .
$$

We denote $X_{i}$ to be the irreducible component (divisor) of $\mathbf{X} \backslash G_{a d}$ that has associated valuation $-\omega_{i}$. The unique closed orbit of $\mathbf{X}$ is $Y=\bigcap_{i=1}^{l} X_{i} \cong(G / B) \times$ $\left(G / B^{-}\right)$.

Moreover, there exists a morphism $\gamma: \mathbb{A}^{l} \rightarrow \mathbf{X}$, where $\mathbb{A}^{l}$ is the affine space of dimension $l$, and an open neighborhood $\mathcal{U} \subset \mathbf{X}$ of $1 \in G_{a d}$ meeting $Y$, such that the map

$$
U \times U^{-} \times \mathbb{A}^{l} \ni\left(u, u^{\prime}, a\right) \mapsto\left(u, u^{\prime}\right) \cdot \gamma(a) \in \mathcal{U}
$$

is an isomorphism. The morphism $\gamma$ verifies that for all $(t, s) \in T_{0} \times T_{0}$, we have $\gamma^{-1}((t, s) \cdot 1)=\left(t^{\alpha_{1}} s^{-\alpha_{1}}, \ldots, t^{\alpha_{1}} s^{-\alpha_{1}}\right)$ (recall that $\alpha_{i}, i=1, \ldots, l$, are the simple roots of $\left.G_{0}\right)$.

Finally, a $G$-embedding has no colors if and only if it equivariantly dominates $\mathbf{X}$ ([8]).

Lemma 1. Let $M$ be a complete $G$-embedding, without colors, and let $Y \subset M$ be a closed $(G \times G)$-orbit. Then $Y \cong G / B \times G / B^{-}$; in particular, $Y$ is separable.

Proof. Consider the canonical $(G \times G)$-equivariant morphism $\pi: M \rightarrow \mathbf{X}$; then $\pi$ is dominant, and as $M$ is complete, $Y \subset \pi^{-1}\left(G / B \times G / B^{-}\right)$. On the other hand, it is known that $Y$ is isomorphic to $(G \times G) / S, S$ a subgroup scheme. It follows that $S \subset B \times B^{-}$. As $Y$ is projective, $S=B \times B^{-}$, and the Lemma is proved.

We recall that if $M$ is a $G$-embedding, then $B \times B^{-}$has a finite number of orbits in $M$. In particular, if $\mathcal{O} \subset M$ is a $(G \times G)$-orbit, then there exists a $\left(B \times B^{-}\right)$-orbit open in $\mathcal{O}$.

Proposition 2. Let $M$ be a normal $G$-embedding, without colors, and let $\mathcal{E}$ be its associated fan. Then there exists an open $\left(B \times B^{-}\right)$-stable subset $\mathcal{W}$ of $M$ isomorphic to $U \times U^{-} \times A$, where $A$ is the toric T-variety associated to $\mathcal{E}$, such that $\mathcal{W}$ intersects every $(G \times G)$-orbit in its open $\left(B \times B^{-}\right)$-orbit.

Proof. Let $\pi: M \rightarrow \mathbf{X}$ be the canonical $(G \times G)$-equivariant morphism and let $\mathcal{U} \subset \mathbf{X}$ be the open subset isomorphic to $U \times U^{-} \times \mathbb{A}^{l}$ constructed for the wonderful embedding. As $\mathcal{U} \cap G_{a d}=U T_{G_{a d}} U^{-}$it follows that $\pi^{-1}(\mathcal{U}) \cap G=U T U^{-}$and that $\mathcal{W}=\pi^{-1}(\mathcal{U}) \cong U \times U^{-} \times A$, where $A=\pi^{-1}\left(\overline{T_{G_{a d}}} \cap \mathcal{U}\right)=\pi^{-1}\left(\mathbb{A}^{l}\right)$. It is clear 
that $A$ is a normal $T$-variety containing $T$ as an open dense set; that is, $A$ is a toric $T$-variety.

Recall that $\mathbf{X} \backslash \mathcal{U}=\bigcup_{i=1}^{l}{\overline{D_{i}}}^{\mathbf{X}}$, where as before $\overline{D_{i}}$ is the color associated to the simple root $\alpha_{i}$ and $-^{\mathbf{X}}$ means that we consider the closure in $\mathbf{X}$. As $\pi\left({\overline{D_{i}}}^{M}\right) \subset$ ${\overline{D_{i}}}^{\mathrm{X}}$, it follows that $\mathcal{W} \subset M \backslash \bigcup_{i=1}^{l}{\overline{D_{i}}}^{M}$. Consider the $(G \times G)$-stable open subset $M_{\mathcal{C}} \subset M$ associated to a cone $\mathcal{C} \in \mathcal{E}\left([\underline{8})\right.$. Then $M_{\mathcal{C}}$ is a $G$-embedding, and

$$
\mathcal{W} \cap M_{\mathcal{C}}=U \times U^{-} \times\left(A \cap M_{\mathcal{C}}\right) \subset M_{\mathcal{C}} \backslash \bigcup_{i=1}^{l}{\overline{D_{i}}}^{M_{\mathcal{C}}} .
$$

As $M$ is without colors, $M_{\mathcal{C}} \backslash \bigcup_{i=1}^{l}{\overline{D_{i}}}^{M_{\mathcal{C}}}$ is the affine open $\left(B \times B^{-}\right)$-stable subset constructed in [8, §3]. Thus, $\pi\left(M_{\mathcal{C}} \backslash \bigcup_{i=1}^{l}{\overline{D_{i}}}^{M_{\mathcal{C}}}\right) \subset \mathcal{U}$. It follows that $\mathcal{W}=M_{\mathcal{C}} \backslash \bigcup_{i=1}^{l}{\overline{D_{i}}}^{M_{\mathcal{C}}}$, so the intersection of $\mathcal{W}$ with a $(G \times G)$-orbit $\mathcal{O}$ of $M$ is the unique open $\left(B \times B^{-}\right)$-orbit of $\mathcal{O}$. Furthermore, as $M_{\mathcal{C}} \cap \mathcal{U}=U \times U^{-} \times\left(A \cap M_{\mathcal{C}}\right)$, it follows that $A_{\mathcal{C}}=A \cap M_{\mathcal{C}}$ is an affine $T$-toric variety.

Recall that $\mathcal{C}_{\mathbb{A}^{l}}$, the cone associated to $\mathbb{A}^{l}$ as a toric variety, is $-C=\mathcal{V}(G)$. If a 1-PS $\lambda$ of $T$ has a limit in $A_{\mathcal{C}}$, then its image by $\pi$ has a limit in $\mathbb{A}^{l}$; thus $\lambda \in \mathcal{V}(G)$. On the other hand, the 1-PS belonging to $\mathcal{V}(G)$ whose limit exists in $M_{\mathcal{C}}$ are exactly those of $\mathcal{V}\left(M_{\mathcal{C}}\right)=\mathcal{C} \cap \mathcal{V}(G)$ (14]). It follows that $\mathcal{C}_{A_{\mathcal{C}}}=\mathcal{C}$, and thus that $\mathcal{E}_{A}=\mathcal{E}$.

Corollary 1. $X$ be a normal $G$-embedding, without colors, with associated fan $\left\{\mathcal{C}_{i}, i \in I\right\}$. Suppose that $\mathcal{C}_{i}$ is generated by a part of a basis of $\Xi(T)$ for all $i \in I$. Then $X$ is smooth.

Proof. Consider the affine chart $\mathcal{W} \cong U \times U^{-} \times A$ of $X$ constructed in Proposition 2]. The fan associated to $A$ as a $T$-toric variety is $\left\{\mathcal{C}_{i}\right\}_{i \in I}$, so $A$ is smooth ([5]), and it follows that $X$ is smooth.

Proposition 3. Let $X$ be a normal $G$-embedding. Then there exist a smooth $G$ embedding $\widetilde{X}$, without colors, and a proper birational $(G \times G)$-equivariant morphism $\varphi: \widetilde{X} \rightarrow X$.

Proof. Let $\mathcal{E}=\left\{\left(\mathcal{C}_{i}(X), \mathcal{F}_{i}(X)\right)\right\}$ be the colored fan associated to $X$, and consider the fan $\mathcal{E}^{\prime}$ obtained by considering the cones $\mathcal{C}_{i}(X) \cap \mathcal{V}(G)$ and their faces. Take a subdivision of $\mathcal{E}^{\prime}$ into a fan $\mathcal{E}^{\prime \prime}$ (necessarily without colors) such that each cone $\mathcal{C}$ of $\mathcal{E}^{\prime \prime}$ is generated by part of a basis of $\Xi(T)$; this subdivision always exists; cf. the toric case ([5]). We claim that the $G$-embedding $\widetilde{X}$ associated to $\mathcal{E}^{\prime \prime}$ verifies the required properties.

Indeed, the subdivision above induces a proper birational equivariant morphism $\varphi: \widetilde{X} \rightarrow X([\underline{8})$, and it follows from Corollary 1 that $\widetilde{X}$ is smooth.

Remark. As the resolution of singularities $\varphi: \tilde{X} \rightarrow X$ constructed above is a birational proper morphism and that $X$ is normal, then $\varphi_{*} \mathcal{O}_{\widetilde{X}}=\mathcal{O}_{X}$. It follows that if $\widetilde{X}$ is Frobenius split, then so is $X$ ([12], [11]).

Proposition 4. Let $X$ be a normal $G$-embedding, without colors. Then a canonical divisor is given by $-\sum_{j=1}^{r} X_{j}-\sum_{i=1}^{l} 2 \overline{D_{i}}$, where $X_{j}, j=1, \ldots, r$, are the $(G \times G)$ stable divisors of $X$ and $D_{i}, i=1, \ldots, l$, are the colors. 
Proof. We identify the $G$-invariant sections of $\mathcal{T} G$, the tangent bundle of $G$, with $\operatorname{Lie}(G)$, the Lie algebra associated to $G$. Consider a basis $\gamma_{1}, \ldots, \gamma_{h}$ of the $U$ left invariants sections of $\mathcal{T} U$, the tangent bundle over $U$, and consider a basis $\gamma_{1}^{\prime}, \ldots, \gamma_{h}^{\prime}$ of the $U^{-}$-right invariant sections of $\mathcal{T} U^{-}$, such that the $\gamma_{i}\left(\right.$ resp. $\left.\gamma_{i}^{\prime}\right)$ correspond to the positive (resp. negative) root vectors of $\operatorname{Lie}(G)$. We choose subscripts in such a way that if $\gamma_{i}$ corresponds to $\alpha, \alpha$ a positive root, then $\gamma_{i}^{\prime}$ corresponds to $-\alpha$. Let $\delta_{1}, \ldots, \delta_{n}$ be a basis of the $(T \times T)$-invariant sections of the tangent bundle of $T$.

As $B B^{-}=U T U^{-}$, we obtain a $\left(B \times B^{-}\right)$-invariant section $0 \neq \sigma \in H^{0}\left(X, \omega_{X}^{-1}\right)$, given by $\sigma=\gamma_{1} \wedge \cdots \wedge \gamma_{h} \wedge \delta_{1} \wedge \cdots \wedge \delta_{n} \wedge \gamma_{1}^{\prime} \wedge \cdots \wedge \gamma_{h}^{\prime} \in H^{0}\left(G, \wedge^{\text {max }} \mathcal{T} X_{\text {reg }}\right)$. Indeed, by construction $\sigma \neq 0$ over the open $\left(B \times B^{-}\right)$-orbit $B B^{-}$. Let us calculate $\operatorname{div}(\sigma)$; as $\sigma$ is $\left(B \times B^{-}\right)$-invariant, we have

$$
\operatorname{div}(\sigma)=\sum_{j=1}^{r} a_{j} X_{j}+\sum_{i=1}^{l} b_{i} \overline{D_{i}}
$$

for some integers $a_{j}, b_{i}$. In order to calculate the order of vanishing of $\sigma$ at $X_{j}$, consider the open chart $U \times U^{-} \times A \hookrightarrow X$ constructed in Proposition 2, It is clear that the vanishing order of $\sigma$ at the divisor $X_{j}$ is given by the vanishing order of the restriction to $A$ of $\delta_{1} \wedge \cdots \wedge \delta_{s}$, which is a section of $\bigwedge^{\text {max }} \mathcal{T} A_{\text {reg }}$. We are reduced to the toric case, and the vanishing order of $\sigma$ at a divisor of the boundary of $A$ is 1 , so $\operatorname{div}(\sigma)=\sum X_{j}+\sum b_{i} \overline{D_{i}}$ ( $\left.(5]\right)$. Indeed, if $\nu_{k}$ is the valuation associated to $X_{k}$, consider $\nu_{i_{1}}, \ldots, \nu_{i_{s}}$ such that $\nu_{j}, \nu_{i_{1}}, \ldots, \nu_{i_{s}}$ generate a cone of maximal dimension belonging to $\mathcal{E}_{A}$. Then in local coordinates the equation of $\sigma$ is $x_{j} x_{i_{1}} \cdots x_{i_{s}}$, so $\sigma$ has vanishing order 1 at $\left\{x_{j}=0\right\}$.

As $X \backslash \bigcup X_{j}=G$, in order to calculate the coefficients $b_{i}, i=1, \ldots l$, we can restrict ourselves to $G$. Recall that the $\left(B \times B^{-}\right)$-stable irreducible divisors in $G$ are the colors $D_{i}=\overline{B s_{\alpha_{i}} B^{-}}=\overline{U s_{\alpha_{i}} T U^{-}}, \alpha_{i}$ a simple root. Consider $\left(\operatorname{ker} \alpha_{i}\right)^{\circ}$, the identity irreducible component of the kernel of the character $\alpha_{i}$, and put $G_{i}=$ $Z_{G}\left(\left(\operatorname{ker} \alpha_{i}\right)^{\circ}\right)$. Let $P_{i}\left(\operatorname{resp} . Q_{i}\right)$ be the minimal parabolic containing $B$ (resp. $B^{-}$) associated to $\alpha_{i}$ (resp. $-\alpha_{i}$ ). From the properties of the Bruhat decomposition we get $B s_{\alpha} B^{-}=P_{i} Q_{i}=R_{u}\left(P_{i}\right) G_{i} R_{u}\left(Q_{i}\right)$, where $R_{u}(H)$ notes the unipotent radical of a group $H$. Recall that $R_{u}\left(P_{i}\right)$ (resp. $R_{u}\left(Q_{i}\right)$ ) is a closed subgroup of $U$ (resp. $U^{-}$). Moreover, $\left\{\gamma_{j}, \gamma_{j} \neq \gamma_{i}\right\}$ (resp. $\left\{\gamma_{j}^{\prime}, \gamma_{j}^{\prime} \neq \gamma_{i}^{\prime}\right\}$ ) is a basis of the $R_{u}\left(P_{i}\right)$-left (resp. $R_{u}\left(Q_{i}\right)$-right) invariant sections of $\mathcal{T} R_{u}\left(P_{i}\right)$ (resp. $\mathcal{T} R_{u}\left(Q_{i}\right)$ ).

From the discussion above it is clear that we can restrict ourselves to $G_{i}$, and then to its semisimple part. This one is isomorphic to $\mathrm{Sl}(2)$ or $\mathrm{PSl}(2)$, and these cases are easily checked. Finally, observe that as the coefficients $a_{j}, b_{i}$ are greater than or equal to zero, it follows that the section $\sigma$ belongs to $H^{0}\left(X, \omega_{X}^{-1}\right)$.

Notation. We denote $\widetilde{G}$ to be the simply connected cover of $G$.

Let $\lambda \in \Xi_{+}(\widetilde{T})$ be a dominant weight of $\widetilde{G}$. We denote $L(\lambda) \rightarrow G / B$ and $L(-\lambda) \rightarrow G / B^{-}$to be the line bundles associated to the weights $\lambda$ (with respect to $B$ ) and $-\lambda$ (with respect to $B^{-}$) respectively $([7])$, and write $H^{0}(G / B, \lambda)=$ $H^{0}(G / B, L(\lambda))$. The external tensor product $L(\lambda) \otimes L(-\lambda) \rightarrow(G / B) \times\left(G / B^{-}\right)$ is denoted $\mathcal{L}(\lambda)$.

Given a dominant weight $\lambda$, there exists a unique line bundle $\mathcal{L}$ over $\mathbf{X}$ such that its restriction to the unique closed orbit $Y$ is $\mathcal{L}(\lambda)([15])$; we denote it as $\mathcal{L}_{\mathbf{X}}(\lambda)$. 
Definition 4. Let $k$ be of characteristic $p>0$, and let $X$ be a $G$-variety. We say that a splitting $\sigma \in H^{0}\left(X, \omega_{X}^{1-p}\right)$ is canonical if

i) $t \cdot \sigma=\sigma$ for all $t \in T$,

ii) for every simple root $\alpha$ there exists $\sigma_{\alpha, r} \in H^{0}\left(X, \omega_{X}^{1-p}\right), r=1, \ldots, p-1$, such that $x_{\alpha}(t) \cdot \sigma=\sum_{r=0}^{p-1} t^{r} \cdot \sigma_{\alpha, r}$.

In order to construct a canonical splitting for an arbitrary normal $G$-embedding, we first recall the construction of a splitting for the wonderful embedding ([2), and show that it is a canonical one.

Definition 5. If $w \in W$ is an element of the Weyl group, we define $\mathbf{X}(w)$, the large Schubert variety, as the closure in $\mathbf{X}$ of the double class $B w B \subset G$.

Notation. From now on, we assume that $\operatorname{char} k=p>0$.

Lemma $\mathbf{2}([2])$. The wonderful embedding $\mathbf{X}$ is canonically split compatibly with all $(G \times G)$-orbit closures and all subvarieties $\mathbf{X}(w)$ and $\left(w_{0}, w_{0}\right) \mathbf{X}(w)$ (obvious action), for $w \in W$ and for $w_{0} \in W$ the element of maximal length.

Proof. In [2] is constructed a splitting $\sigma \in H^{0}\left(\mathbf{X}, \omega_{\mathbf{X}}^{1-p}\right)$ verifying all but the first assertion. We recall the construction given there in order to prove that $\sigma$ is a canonical splitting.

Consider the Steinberg module $S t=H^{0}(G / B,(p-1) \rho)$. Using Frobenius reciprocity and selfduality of $S t$ we obtain a $(\widetilde{G} \times \widetilde{G})$-homomorhism

$$
f: S t \otimes S t \rightarrow H^{0}\left(\mathbf{X}, \mathcal{L}_{\mathbf{X}}((p-1) \rho)\right),
$$

such that the composition

$$
r e s_{Y} \circ f: S t \otimes S t \rightarrow H^{0}(Y, \mathcal{L}((p-1) \rho))
$$

is an isomorphism, where $Y$ denotes as usual the unique closed orbit of $X$.

Consider the $(\widetilde{G} \times \widetilde{G})$-morphism

$$
\begin{aligned}
f^{2}:(S t \otimes S t)^{\otimes 2} & \rightarrow H^{0}\left(\mathbf{X}, \mathcal{L}_{\mathbf{X}}(2(p-1) \rho)\right), \\
x_{1} \otimes y_{1} \otimes x_{2} \otimes y_{2} & \mapsto f\left(x_{1} \nabla y_{1}\right) f\left(x_{2} \otimes y_{2}\right) .
\end{aligned}
$$

The composition $\operatorname{res}_{Y} \circ f^{2}:(S t \otimes S t)^{\otimes 2} \rightarrow H^{0}(Y, \mathcal{L}((p-1) \rho))$ is surjective. As $\operatorname{Hom}\left(F_{*} \mathcal{O}_{Y}, \mathcal{O}_{Y}\right)$ is isomorphic to $H^{0}(Y, \mathcal{L}((p-1) \rho))$, we obtain an unique homomorphism (up to a constant)

$$
\varphi:(S t \otimes S t)^{\otimes 2} \rightarrow \operatorname{Hom}\left(F_{*} \mathcal{O}_{Y}, \mathcal{O}_{Y}\right) .
$$

Let $v_{+}$(resp. $v_{-}$) be a highest (resp. lowest) weight vector in $H^{0}(G / B, \rho)$. Then $a=\left(v_{+} \otimes v_{+}\right)^{p-1}$ and $b=\left(v_{-} \otimes v_{-}\right)^{p-1}$ are in $S t \otimes S t$ and are such that $\varphi(a \otimes b)$ is a splitting of $Y$.

By the proof of [16, Prop. 4.3.17], $a \otimes b$ verifies the conditions i) and ii) of the definition of canonical splitting. As $f^{2}$ is $(\widetilde{G} \times \widetilde{G})$-equivariant, it follows that $f^{2}(a \otimes b)$, and thus $\tau=\varphi(a \otimes b)$ satisfy the conditions, too. Write $x_{\alpha}(t) \cdot \tau=$ $\sum_{r=0}^{p-1} t^{r} \cdot \tau_{\alpha, r}$, with $\tau_{\alpha, r} \in H^{0}\left(\mathbf{X}, \mathcal{L}_{\mathbf{X}}(2(p-1) \rho)\right)$.

Consider the $(\widetilde{G} \times \widetilde{G})$-invariant sections $\sigma_{i}$ of $\mathcal{L}_{X}\left(\alpha_{i}\right)=\mathcal{O}_{X}\left(-X_{i}\right), i=1, \ldots, l$; then the section

$$
\sigma=\tau \prod \sigma_{i}^{p-1}
$$


is the splitting we search for. Indeed, it is a Frobenius splitting ([2]), and if $t \in$ $T \times T$, then

$$
t \cdot \sigma=t \cdot \tau \prod_{i=1}^{l}\left(t \cdot \sigma_{i}\right)^{p-1}=\tau \prod \sigma_{i}^{p-1}
$$

because $\sigma_{i}$ is $(G \times G)$-invariant for $i=1, \ldots, l$. Moreover, if $\alpha$ is a simple root of $G \times G$, then

$$
x_{\alpha}(t) \cdot \sigma=\left(\sum_{r=0}^{p-1} t^{r} \cdot \tau_{\alpha, r}\right) \prod \sigma_{i}^{p-1}=\sum_{r=0}^{p-1} t^{r} \cdot\left(\tau_{\alpha, r} \prod \sigma_{i}^{p-1}\right),
$$

and the proposition is proved.

Theorem 2. Any normal G-embedding is canonically Frobenius split.

Proof. Let $M$ be a normal $G$-embedding. Consider the resolution of singularities $\varphi: \widetilde{M} \rightarrow M$ constructed in Proposition 3 A (canonically) Frobenius splitting for $\widetilde{M}$ induces a (canonical) Frobenius splitting for $M$, so we can assume that $M$ is smooth, without colors. If we consider a smooth completion $M \hookrightarrow \bar{M}$ of $M$, then if $\bar{M}$ is (canonical) Frobenius split so is $M$, thus we suppose $M$ without colors, smooth and complete.

By Proposition 4 a canonical divisor for $M$ is given by $\sum_{i=1}^{l}-2 \overline{D_{i}}-\sum_{j=1}^{r} X_{j}$, where $\overline{D_{i}}$ is the closure of the color associated to the simple root $\alpha_{i}, i=1, \ldots, l$, and $X_{j}, j=1, \ldots, r$, are the $(G \times G)$-stable irreducible divisors.

Let $Y \cong G / B \times G / B^{-}$be a closed orbit of $M$. Then $Y$ is Frobenius split ([11],[15],[2]), and $\omega_{Y} \cong \mathcal{L}(-2 \rho)$, where $\rho$ is the half sum of the positive roots. As $M$ is smooth, from the proof of Proposition 3 we get that a cone of maximal dimension of the colored fan associated to $M$ must be generated by a basis of $\Xi(T)$. We change subscripts of the $(G \times G)$-stable divisors $X_{j}, j=1, \ldots, r$, in such a way that $Y$ is a transversal intersection of the divisors $X_{j}, j=1, \ldots, n$, where $n=\operatorname{dim} T$.

Consider $\varphi: M \rightarrow \mathbf{X}$, the canonical $(G \times G)$-equivariant morphism. As $M$ and $\mathbf{X}$ are without colors, $\varphi^{-1}\left(\bigcup{\overline{D_{i}}}^{\mathbf{X}}\right)=\bigcup{\overline{D_{i}}}^{M}$. It follows that $\varphi^{*} \mathcal{O}\left(\sum{\overline{D_{i}}}^{\mathbf{X}}\right)=$ $\mathcal{O}\left(\sum{\overline{D_{i}}}^{M}\right)$. By the projection formula, we have

$$
\begin{aligned}
\varphi_{*} \mathcal{O}\left(2(p-1) \sum{\overline{D_{i}}}^{M}\right) & =\varphi_{*} \varphi^{*} \mathcal{O}\left(2(p-1) \sum{\overline{D_{i}}}^{\mathbf{X}}\right) \\
& =\varphi_{*}\left(\mathcal{O}_{M}\right) \otimes_{\mathcal{O}_{\mathbf{X}}} \mathcal{O}\left(2(p-1) \sum{\overline{D_{i}}}^{\mathbf{X}}\right) .
\end{aligned}
$$

So, if the section $\sigma$ is as in the proof of Lemma 2, we can think of $\sigma$ as belonging to $H^{0}\left(\mathbf{X}, \varphi_{*} \mathcal{O}\left(2(p-1) \sum \overline{D_{i}}\right)\right)=H^{0}\left(M, \mathcal{O}\left(2(p-1) \sum \overline{D_{i}}\right)\right)$.

Take $\widetilde{\sigma}=\sigma \prod_{j=1}^{r} \sigma_{j}^{p-1}, \sigma_{j}$ a $(G \times G)$-invariant section for $\mathcal{O}\left(X_{j}\right)$; then $\widetilde{\sigma} \in$ $H^{0}\left(M, \omega_{M}^{1-p}\right)$. We take as coordinates in a neighbourhood of the point $x_{0}$ the system $y_{1}, \ldots, y_{m}, s_{1}=\sigma_{1}, \ldots, s_{n}=\sigma_{n}$. It follows that the expansion in these coordinates of $\sigma$ has a non-zero coefficient at the point $x_{0}$ for the term $(y s / d y d s)^{p-1}$, so $M$ is Frobenius split ([11]).

Moreover, it is clear from the construction that the section $\widetilde{\sigma}$ constructed gives a canonical splitting (using the same calculations as in Lemma 2).

Corollary 2. In arbitrary characteristic, any normal G-embedding has a rational resolution. In particular, it is a Cohen-Macaulay variety. 
Proof. Suppose char $k=p>0$, and let $\pi: \widetilde{X} \rightarrow X$ be the desingularisation of a normal $G$-embedding $X$ constructed in Proposition 3 It is clear that the exceptional locus of $\pi$ is included set theoretically in the union $\bigcup_{j=1}^{r} X_{j}=X \backslash G$ of the $(G \times G)$-stable irreducible divisors. The divisor associated to the section $\sigma \in H^{0}\left(X, \omega_{X}^{1-p}\right)$ constructed in the proof of Theorem 2 contains this union, so we are in conditions to apply the results of [1, §3], and deduce that $X$ has a rational resolution. The case char $k=0$ is obtained by reduction $\bmod p([1])$.

\section{Good FiLtrations}

Let $M$ be a (normal, irreducible) reductive monoid with unit group $G$, such that the center of $G$ is one-dimensional. In [3, it is proved that in that case the algebra of regular functions of $M$ has a good filtration for the induced action of $G \times G$. We extend this result to arbitrary (normal, irreducible) reductive monoids, as a consequence of the existence of a canonical Frobenius splitting for $M$.

Definition 6. Let $G$ be a reductive group; a rational $G$-module $V$ has a good filtration if there exists a filtration

$$
(0)=V_{0} \subset V_{1} \subset \cdots
$$

such that $\bigcup V_{i}=V$ and for all $i$ there exists $\lambda_{i} \in \Xi^{+}(T)$ such that $V_{i+1} / V_{i} \cong$ $H^{0}\left(G / B, \lambda_{i}\right)$, the highest weight module of greatest weight $\lambda_{i}$.

Corollary 3. Let $M$ be a reductive monoid. Then $k[M]$, algebra of regular functions of $M$, has a good filtration for the action of $G \times G$ induced by the action on $M$.

Proof. As $M$ admits a canonical Frobenius splitting, it follows from [10, Thm. 6.2] that the $(G \times G)$-module $H^{0}(M, \mathcal{L})$ has a good filtration for all $(G \times G)$-equivariant line bundles $\mathcal{L}$. In particular $k[M]=H^{0}\left(M, \mathcal{O}_{M}\right)$ has a good filtration.

\section{REFERENCES}

[1] M. Brion and S.P. Inamdar, Frobenius splitting of spherical varieties. Proc. of Symp. in Pure Math. 56 (1994) Part I, pages 207-218. MR 95e:14037

[2] M. Brion and P. Polo, Large Schubert varieties. Representation Theory 4 (2000), pages 97-126. MR 2001j:14066

[3] S. Doty, Representation theory of reductive normal algebraic monoids. Transactions of the A.M.S. 356 N. 6 (1999), pages 2539-2551. MR 99i:20086

[4] C. De Concini and C. Procesi, Complete symmetric varieties. In M.F. Gherardelli, ed. Invariant Theory, Proceedings. Lect. Notes in Math. 996, pages 1-44. Springer-Verlag, 1983, New York. MR 85e:14070

[5] W. Fulton, Introduction to Toric Varieties, Princeton Univ. Press, 1993. MR 94g:14028

[6] R. Hartshorne, Algebraic Geometry, GTM 52, Springer-Verlag, 1977. MR 57:3116

[7] J.C. Jantzen, Representations of algebraic groups. Academic Press, 1987. MR 89c:20001

[8] F. Knop, The Luna-Vust theory of spherical embeddings. In S. Ramanan et al., editors, Proceedings of the Hydebarad Conference on Algebraic Groups, pages 225-249. National Board for Higher Mathematics, Manoj, 1991. MR 92m:14065

[9] N. Lauritzen, Splitting properties of complete homogeneous spaces. J. Algebra 162 (1993), no. 1, pages 178-193. MR 95f:14095

[10] O. Mathieu, Tilting modules and thier applications, Adv. Studies in Pure Math. 26 (2000), pages 1-68. MR 2001k:20095

[11] V.B. Mehta and A. Ramanathan, Frobenius splitting and cohomology vanishing for Schubert varieties, Ann. of Math. (2) 122 (1985), pages 27-40. MR 86 k:14038

[12] A. Ramanathan, Equations defining Schubert varieties and Frobenius splitting of diagonals. Publ. Math. IHES 65 (1987), pages 61-90. MR 88k:14032 
[13] L. Renner, Cohen-Macaulay algebraic monoids. Proc. of the Amer. Math. Soc. 89, N. 4, December 1983, pages 574-578. MR 85c:20063

[14] A. Rittatore, Algebraic monoids and group embeddings. Trans. Groups Vol. 3, No. 4 (1998), pages 375-396. MR 2000a:14056

[15] E. Strickland, A vanishing theorem for group compactifications. Math. Ann. 277 (1987), pages 165-171. MR 88b:14035

[16] W. Van der Kallen, Frobenius splittings and B-modules. Springer-Verlag, 1993. MR 95i:20064

[17] E.B. Vinberg, On reductive algebraic semigroups. Amer. Math. Soc. Transl., Serie 2, 169 (1994), pages 145 - 182. Lie Groups and Lie Algebras. E.B. Dynkin seminar. MR 97d:20057

[18] T. Vust, Plongements d'espaces symétriques algébriques: une classification. Ann. Scuola Norm. Sup. Pisa, 17, 2 (1990), pages 165-194. MR 91m:14079

Facultad de Ciencias, Universidad de la República, Iguá 4225, 11400 Montevideo, URUGUAY

E-mail address: alvaro@cmat.edu.uy 